\title{
Schroederella berkeleyi sp.n., un nouvel Orbiniidé (Polychète Sédentaire) de la faune interstitielle littorale du Massachusetts (USA) ${ }^{1}$
}

\author{
LUCIEN LAUBIER \\ Centre Océanologique de Bretagne \\ B.P. 337, Brest 29N., France
}

LAUBIER, L. 1971. Schroederella berkeleyi sp.n., un nouvel Orbiniidé (Polychète Sédentaire) de la faune interstitielle littorale du Massachusetts (USA). J. Fish. Res. Bd. Canada 28: $1483-1486$.

- L'auteur décrit une nouvelle espèce d'annélides polychètes Orbiniidae du genre Schroederel$l a$, et la compare à l'unique espèce antérieurement connue, $S$. pauliani. Comme le générotype, $S$. berkeleyi sp.n. habite le domaine interstitiel littoral, et s'en distingue du double point de vue morphologique et biogéographique. -

LAUBIER, L. 1971. Schroederella berkeleyi sp.n., un nouvel Orbiniidé (Polychète Sédentaire) de la faune interstitielle littorale du iMassachusetts (USA). J. Fish. Res. Bd. Canada 28: $1483-1486$.

- The author describes a new species of polychaetous annelid belonging to the genus Schroederella (Orbiniidae), and compares it to the only previously known species, $S$. pauliani. Like the type-species, $S$. berkeleyi sp.n. lives in the littoral interstitial biotope, and is distinguishable from it by both morphological and biogeographical arguments.

Received May 6, 1971

Parmi les annélides polychètes adaptées à la vie dans le domaine interstitiel littoral (mesopsammon de Remane et Schulz 1934), la famille des Orbiniidae Hartman 1942 (=Ariciidae Audouin et Milne Edwards 1833) ne comprenait jusqu'à présent qu'un seul genre strictement inféodé au milieu interstitiel, le genre Schroederella Laubier 1962, récolté dans les sables littoraux de Sandwich Bay, au Sud de Walvis Bay, en Afrique du Sud (Laubier 1962, 1967). Ce genre, créé pour renfermer l'unique espèce Schroederella pauliani Laubier 1962, est demeuré jusqu'à présent monotypique.

C'est donc avec un grand intérêt que j'ai recu une trentaine de Polychètes interstitielles récoltées sur les côtes du Massachusetts (USA) que le Dr M. Pettibone, qui avait reconnu leur appartenance au genre Schroederella, a bien voulu m'adresser pour étude. L'examen de ces spécimens a montré qu'ils différaient spécifiquement du générotype

${ }^{1}$ Contribution no 51 du Groupe Scientifique du Centre Océanologique de Bretagne.

This issue of the Journal of the Fisheries Research Board of Canada is dedicated to Dr Cyril Berkeley and to the memory of the late Mrs Edith Berkeley.

Printed in Canada (J2162)
Schroederella pauliani, comme le laissait prévoir l'éloignement des deux localités. L'espèce nouvelle est sincèrement dédiée au Dr C. Berkeley.

\section{Schroederella berkeleyi sp.n.}

\section{Fig. 1}

Localité, types-Trente spécimens de $S$. berkeleyi sp.n. ont été récoltés dans les sables intertidaux de Craigville Beach à Centerville, Barnstable County, Massachusetts, par W. J. Wall, le 26 juin 1969. La zone de récolte est située dans une petite baie où débouche une rivière (Bumps River); la salinité de l'eau y varie entre $26 \%$ à marée basse et $31 \%$ à marée haute. Le sable est riche en particules organiques. Ce matériel a été déposé dans les collections de la Smithsonian Institution sous les numéros de catalogue 44946 pour l'holotype et 44947 pour les paratypes.

Taille, coloration- La longueur de $S$. berkeleyi sp.n. varie entre les deux valeurs extrêmes de $2.5 \mathrm{~mm}$ (individu de 22 segments) et $6.6 \mathrm{~mm}$ (individu de 40 segments). La valeur la plus fréquente est de 6 à 6.2 $\mathrm{mm}$, avec un nombre total de segments compris entre 30 et 35 . Des chiffres du même ordre ont été relevés chez $S$. pauliani. Toutefois, chez cette dernière espèce, des individus de plus petite taille avaient été récoltés: 1 $\mathrm{mm}$ de longueur pour 8 a 10 segments. 
A
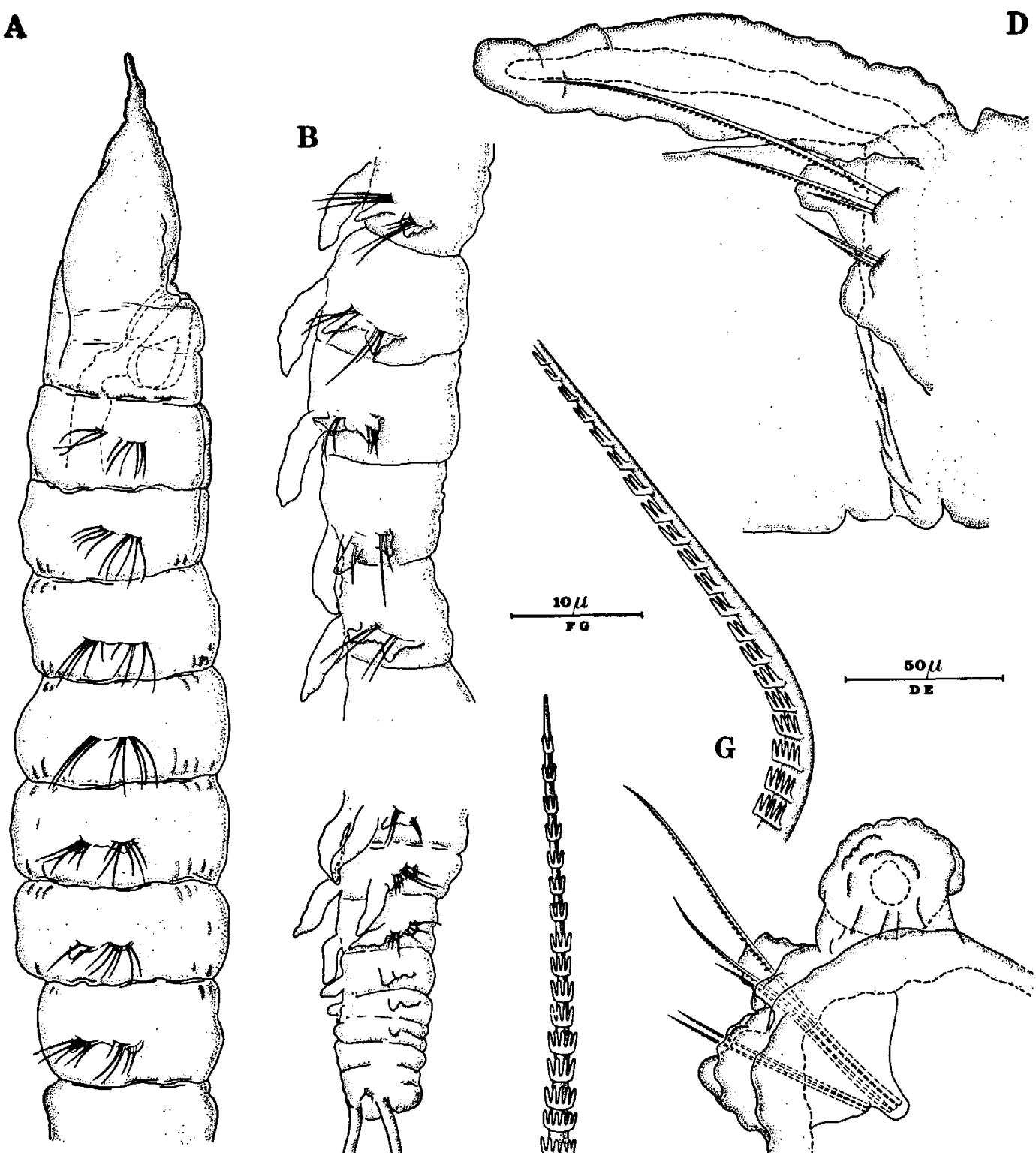

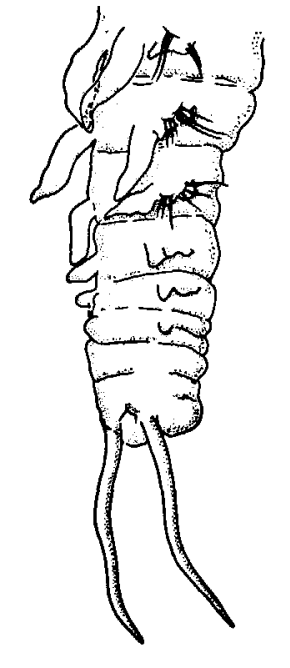

C

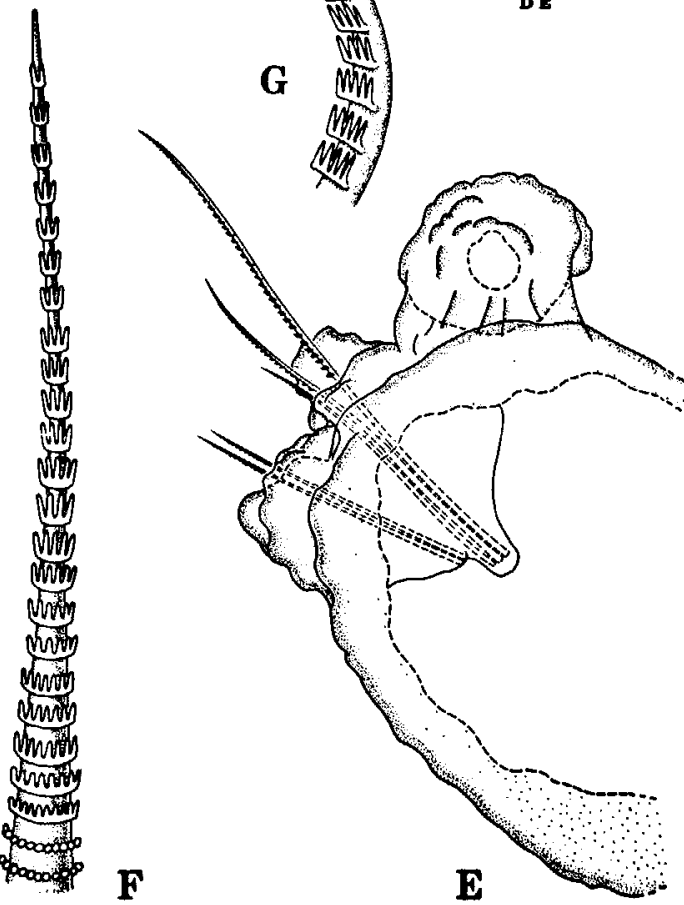

Fig. 1. Schroederella berkeleyi sp.n. holotype: A, partie antérieure; B, région abdominale, du 24ème au 28 ème segment; $C$, région abdominale, du 30ème segment au pygidium; $D$, 28ème segment en vue latérale; $E$, le même vu de face; $F$, soie à plaquettes crénelées de face; $G$, la même de profil. 
Dans l'alcool, les animaux sont blanchâtres; seules sont visibles les deux taches rouge vermillon des yeux. Une dépigmentation semblable avait été observée chez $S$. pauliani; elle est probablement en relation avec le mode de vie de ces animaux.

Prostomium - Le prostomium de $S$. berkeleyi sp.n. est semblable à celui de $S$. pauliani. Le prolongement antérieur régulièrement effilé est une caractéristique générique, en même temps qu'une remarquable adaptation à la vie dans le domaine interstitiel. Cependant, l'articulation entre la pointe et la partie postérieure du prostomium est moins marquée que chez $S$. pauliani. Les deux yeux rouge vermillon sont profondément enfoncés dans les téguments, et les organes nucaux décrits chez $S$. pauliani ne sont pas visibles chez $S$. berkeleyi sp.n.

Corps - Le corps est divisé en deux régions, une région thoracique abranche et une région abdominale branchifère. La transition entre ces deux parties est progressive, la branchic apparaissant d'abord sous la forme d'une protubérance hémisphérique, puis d'un prolongement de plus en plus long à extrémité arrondie. Cette zone de transition compte 3 à 5 segments. La première branchic (c'est-d-dire au début de cette zone de transition) apparaít au 14-15ème segment (soit le 12e-13e sétigère) chez les animaux de grande taille, au 1le segment (soit le 9e sétigère) chez le plus petit individu.

Le segment buccal est moins distinct que chez $S$. pauliani. Cependant, il existe bien deux segments achètes en arrière du prostomium, comme le laisse supposer la longueur de l'ensemble compris entre ce dernier et le premier segment sétigère: on distingue une très fine ligne intersegmentaire.

Région thoracique - Les parapodes de cette région présents à partir du 3ème segment comprennent deux rames, un notopode et un neuropode. Les lobes postérieurs apparaissent à la rame dorsale au 7 ème segment, sous forme d'un simple bouton qui s'accroit ensuite sur les segments postérieurs pour former un prolongement digitiforme. A la rame ventrale, il n'y a pas de lobe postérieur, mais seulement un mamelon sétigère qui devient bien visible à partir du 7ème segment également.

Dans la région thoracique, les soies sont toutes semblables. Ce sont des soies capillaires à plaquettes crénelées, identiques à celles décrites chez $S$. pauliani. Ces plaquettes sont constituées par des anneaux incomplets insérés à peu près perpendiculairement au corps de la soie et porteurs d'une série de 12 à 2 fines dents parallèles à l'axe de la soie, le nombre de dents décroissant de la base vers la pointe de la soie. Cette structure, délicate à observer, est semblable à celle décrite chez $S$. pauliani. Le nombre de soies à plaquettes crénelées décroît d'avant en arrière: de 8 à 9 soies à chacune des deux rames des segments les plus antérieurs, à 2 à 3 soies dans les derniers segments antépygidiaux.
Chaque rame est soutenue par un (parfois deux) acicule pointu. Contrairement à ce qui se passe chez $S$. pauliani, il n'y a jamais d'uncini sur les neuropodes de la région thoracique. A elle seule, cette différence justifie la création de l'espèce nouvelle $S$. berkeleyi.

Région abdominale - Cette région est essentiellement caractérisée par l'apparition des branchies. Les branchies, en position latéro-dorsale, sont constituées par un simple filament inséré au niveau du parapode, recourbé vers l'arrière et prenant très tôt une direction à peu près parallèle à l'axe longitudinal du corps. Dans la région abdominale, le lobe dorsal est bien développé, et représente environ le cinquième de la longueur de la branchie. Il n'y a pas de véritable lobe postsétal au neuropode, mais un simple mamelon sétigère. Aux deux rames, les soies capillaires à plaquettes crénelées sont toujours présentes, parfois accompagnées aux notopodes d'une soie capillaire lisse en aiguille, plus fine que les soies a plaquettes. Il n'y a pas d'uncini dans la région abdominale, pas plus que dans la région thoracique. Chaque rame est soutenue par un acicule en aiguille. Enfin, on distingue parfois de très petites soies capillaires, qui semblent bien, d'après leur ornementation, n'être que des soies à plaquettes en formation.

Les trois à quatre derniers segments antépygidiaux sont dépourvus de soies, on distingue simplement les ébauches des branchies et des parapodes.

Pygidium - L'anus s'ouvre dorsalement, entre les bases de deux longs et grêles cirres latéro-dorsaux. Ventralement, le pygidium porte deux lobes trapus et grossièrement arrondis. A la longueur des cirres latérodorsaux près, tout ceci est semblable au pygidium de S. pauliani.

\section{Justification}

Sur le plan morphologique, les différences entre $S$. pauliani et $S$. berkeleyi sp.n., bien que peu nombreuses, paraissent suffisamment importantes pour justifier la création d'une espèce nouvelle pour la forme américaine. $S$. berkeleyi sp.n. se distingue du générotype par l'absence complète d'uncini, la longueur exceptionnelle des cirres pygidiaux latéro-dorsaux, la segmentation peu marquée entre prostomium et premier segment et premier et second segments, enfin l'articulation indistincte de la partie antérieure du prostomium avec la base. En plus de ces critères morphologiques, la répartition des deux espèces apporte un élément important du point de vue biogéographique, même si l'on tient compte du cosmopolitisme relatif (c'est-à-dire considéré par rapport aux formes voisines non interstitielles) des animaux peuplant les sables intertidaux: $S$. pauliani, décrite de la région de Walvis Bay (Sud-Est Atlantique), n'a jamais été retrouvée depuis sa découverte (Day 1967), et $S$. berkeleyi sp.n. a été recueillie sur les 
côtes du Massachusetts (Nord-Ouest Atlantique).

Depuis la création du genre Schroederella, la sous-famille des Protoariciinae Hartman 1957 s'est enrichie de deux genres nouveaux non interstitiels, Protoariciella Hartmann-Schröder 1962 et Scoloplella Day 1963. Le prostomium normalement arrondi de ces deux genres, les biotopes respectifs des trois espèces qu'ils comprennent actuellement, bien différents des sables intertidaux, confirment la validité du genre Schroederella.

DAY, J. H. 1963. The Polychaete fauna of South Africa Pt. 8: new species and records from grab samples and dredgings. Bull. Brit. Mus. (Natur. Hist.) Zool. 10: 381-445.

1967. Sedentaria, p. 459-878. In A monograph on the Polychaeta of southern Africa. Pt. 2. Trustees British Mus. (Natur. Hist.), London.
Hartman, O. 1942. A review of the types of polychaetous annelids at the Peabody Museum of Natural History, Yale University. Bull. Bingham Oceanogr. Collect. Yale Univ. 8: 1-98.

HARTMANN-SChröDeR, G. 1962. Zweiter Beitrag zur Polychaetenfauna von Peru. Kiel. Meeresforsch. 18(1): $109-147$.

LAUBIER, L. 1962. Schroederella pauliani gen.nov., sp.nov., un nouvel Orbiniidé (polychètes sédentaires) de la faune interstitielle d'Afrique. Ann. Transv. Mus. 24 ( 2 et 3 ): 231-238.

1967. Adaptations chez les annélides polychètes interstitielles. Ann. Biol. 6(1-2): 1-16.

Remane, A., et E. Schulz. 1934. Die Tierwelt des Küstengrundwassers sei Schilksee (Kieler Bucht) I-VII-I. Das Küstengrundwasser als Lebensraum. Schr. Naturwiss. Ver. Schleswig-Holstein 20: 399408. 